\title{
A method of lines approach for modelling saltwater intrusion in coastal aquifers
}

\author{
$\begin{array}{ll}\text { T. J. Moroney } & \text { S. L. Truscott } \\ & \end{array}$
}

(Received 15 August 2008; revised 8 December 2008)

\begin{abstract}
The equations governing saltwater intrusion in coastal aquifers are complex. Backward Euler time stepping approaches are often used to advance the solution to these equations in time, which typically requires that small time steps be taken in order to ensure that an accurate solution is obtained. We show that a method of lines approach incorporating variable order backward differentiation formulas can greatly improve the efficiency of the time stepping process.
\end{abstract}

\section{Contents}

\section{Introduction}

http://anziamj . austms.org. au/ojs/index.php/ANZIAMJ/article/view/1413 gives this article, (c) Austral. Mathematical Soc. 2008. Published December 23, 2008. ISSN 1446-8735. (Print two pages per sheet of paper.) 
2 Mathematical model

C627

3 Numerical solution

C628

3.1 Spatial discretisation . . . . . . . . . . . . . C628

3.2 Temporal discretisation . . . . . . . . . . . . . C630

4 Results

C631

4.1 Test problem ................... . C631

4.2 Bundaberg problem ................. C634

5 Conclusion

C635

References

C637

\section{Introduction}

Groundwater aquifer systems have for a long time been used to provide fresh water for irrigation and other purposes in many parts of the world. Careful management of these valuable resources is essential to ensure that they are not overused. In the case of coastal aquifers, an additional and important complication is the potential for saltwater intrusion if the aquifers are stretched beyond their long term yield [1].

In an attempt to better understand and ultimately predict future behaviours of these aquifer systems, mathematical models have been developed to describe the process of saltwater intrusion $[4,6,7,9]$. The mechanisms governing this process are complex, involving variable density fluid flow in anisotropic, heterogeneous porous media.

Obtaining accurate solutions to these equations can be costly in terms of computational work required. In their investigation of Richards' equation in one spatial dimension, Tocci et al. found that a method of lines approach 
incorporating variable order, backward differentiation formulas substantially reduced the overall cost of solution compared to traditional backward Euler time stepping approaches [8]. We demonstrate that a similar approach, based on a finite volume spatial discretisation, is effective at solving the coupled, nonlinear system of PDEs that arises in the modelling of saltwater intrusion in groundwater aquifer systems.

\section{Mathematical model}

The primary variables in the model are reference hydraulic head and salt concentration, denoted by $h$ and $C$ respectively. The fluid density $\rho$ depends on concentration, and is modelled using

$$
\rho=\rho_{0}(1+\eta C),
$$

where $\eta=\varepsilon / C_{s}$ is the density coupling coefficient, $\varepsilon=\left(\rho_{s}-\rho_{0}\right) / \rho_{0}$ is the density difference ratio, $C_{s}$ is the salt concentration of seawater and $\rho_{0}$ and $\rho_{s}$ are the densities of freshwater and seawater respectively.

The average fluid velocity, $\mathbf{v}$, is given by Darcy's law [6]

$$
\mathbf{v}=-\frac{\mathbf{k} \rho_{\circ} \mathrm{g}}{\phi \mu}(\nabla \mathrm{h}+\eta \mathrm{C} \nabla z),
$$

where $\mathbf{k}$ is the permeability tensor, $\mathbf{g}$ is the gravitational acceleration, $\phi$ is the porosity and $\mu$ is the dynamic viscosity.

The specific storage of the aquifer is $S_{s}=\rho g[(1-\phi) \alpha+\phi \beta]$, where $\alpha$ is the porous matrix compressibility and $\beta$ is the fluid compressibility. The pumping rate per unit volume of the aquifer is denoted by $q$.

The dispersion tensor $\mathbf{D}$ takes the following form [2]

$$
\mathbf{D}=\left(\alpha_{\mathrm{L}}-\alpha_{\mathrm{T}}\right) \frac{\mathbf{v} \mathbf{v}^{\mathrm{T}}}{\|\mathbf{v}\|}+\left(\alpha_{\mathrm{T}}\|\mathbf{v}\|+\mathrm{D}_{\mathrm{m}}\right) \mathbf{I},
$$


where $D_{m}$ is the molecular diffusivity and $\alpha_{L}$ and $\alpha_{T}$ are the longitudinal and transverse dispersivities respectively.

With the above relations in place, the following two partial differential equations model the saltwater intrusion problem [6]

$$
\begin{aligned}
\rho_{0} S_{s} \frac{\partial h}{\partial t}+\phi \rho_{0} \eta \frac{\partial C}{\partial t}+\nabla \cdot(\phi \rho \mathbf{v}) & =\rho q \\
\phi \frac{\partial C}{\partial t}+\nabla \cdot(\phi C \mathbf{v}) & =\nabla \cdot(\phi D \nabla C)+C q .
\end{aligned}
$$

Equation (1) describes the flow of a variable density fluid, while (2) describes the transport of dissolved salt. Initial and boundary conditions for these equations are discussed in Section 4.

\section{Numerical solution}

\subsection{Spatial discretisation}

Following Liu et al. [7], we discretise in space using a vertex centred finite volume method over a rectangular mesh. Around each node is constructed a rectangular control volume, such that no two control volumes intersect, and their union is the entire domain.

Integrating (1) and (2) over a control volume $\boldsymbol{V}_{\boldsymbol{i}}$, we obtain

$$
\begin{aligned}
& \int_{V_{i}} \rho_{0} S_{s} \frac{\partial h}{\partial t} d V+\int_{V_{i}} \phi \rho_{0} \eta \frac{\partial C}{\partial t} d V+\int_{V_{i}} \nabla \cdot(\phi \rho \mathbf{v}) d V=\int_{V_{i}} \rho q d V \\
& \int_{V_{i}} \phi \frac{\partial C}{\partial t} d V+\int_{V_{i}} \nabla \cdot(\phi C V) d V=\int_{V_{i}} \nabla \cdot(\phi D \nabla C) d V+\int_{V_{i}} C q d V .
\end{aligned}
$$


For flux terms we apply the divergence theorem, while for all other terms we introduce control volume averaged quantities, denoted with an overline

$$
\begin{aligned}
& \rho_{0}\left(\overline{S_{s} \frac{\partial h}{\partial t}}\right)_{i} \Delta V_{i}+\phi \rho_{0} \eta\left(\overline{\frac{\partial C}{\partial t}}\right)_{i} \Delta V_{i}+\iint_{\partial V_{i}}(\phi \rho \mathbf{v}) \cdot \hat{\mathbf{n}} \mathrm{d} \sigma=(\overline{\rho q})_{i} \Delta V_{i}, \\
& \phi\left(\overline{\frac{\partial C}{\partial t}}\right)_{i} \Delta V_{i}+\iint_{\partial V_{i}}(\phi C \mathbf{v}) \cdot \hat{n} d \sigma=\iint_{\partial V_{i}}(\phi D \nabla C) \cdot \hat{n} d \sigma+(\overline{C q})_{i} \Delta V_{i}
\end{aligned}
$$

Equations (5) and (6) are exact restatements of (1) and (2) in control volume form. The approximation comes when control volume averaged quantities are replaced by their values at the corresponding node, to obtain

$$
\begin{aligned}
& \rho_{0}\left(S_{s}\right)_{i} \frac{d h_{i}}{d t}+\phi \rho_{0} \eta \frac{d C_{i}}{d t}=\frac{1}{\Delta V_{i}} \iint_{\partial V_{i}}(-\phi \rho \mathbf{v}) \cdot \hat{\mathbf{n}} d \sigma+(\rho q)_{i}, \\
& \phi \frac{d C_{i}}{d t}=\frac{1}{\Delta V_{i}} \iint_{\partial V_{i}}(-\phi C \mathbf{v}+\phi D \nabla C) \cdot \hat{n} d \sigma+(C q)_{i} .
\end{aligned}
$$

This approximation is second order in space provided the nodes coincide with the control volume centroids. To approximate the surface integrals, we use a midpoint quadrature rule, along with standard bilinear finite element shape functions to evaluate the fluxes at the control volume faces. This approach is sometimes known as the control volume, finite element method (or CVFEM). Upstream weighting handles the advection terms, as was done by Liu et al. [7].

To facilitate parallelism in the code, the mesh is partitioned into nonoverlapping subdomains, and each is assigned to a separate MPI process. One layer of neighbouring node (or 'ghost node') information is required to evaluate the fluxes in (7) and (8); the subdomains are otherwise independent. 


\subsection{Temporal discretisation}

To advance the solution in time we use the backward differentiation formulas (BDFs) provided by the Suite of Nonlinear and Differential/Algebraic Equation Solvers (SUNDIALS) [5], and in particular the Implicit Differential/ Algebraic (IDA) solver therein. This solver uses variable order, variable coefficient BDFs in fixed-leading-coefficient form [3]

$$
\sum_{i=0}^{p} \alpha_{n, i} \mathbf{y}^{(n-i)}=\delta t_{n} \dot{\mathbf{y}}^{(n)},
$$

where $\mathbf{y}^{(\mathfrak{n})}$ and $\dot{\mathbf{y}}^{(\mathfrak{n})}$ are the computed values of the dependent variable and its derivative at time step $n, \delta t_{n}$ is the time step size and $p$ is the order of the method (up to a maximum order of five). In an attempt to achieve optimal efficiency of the method, IDA dynamically adjusts both $\delta t_{n}$ and $p$ at each iteration by analysing local truncation error estimates [5].

Applying (9) to (7) and (8), where

$$
y_{i}= \begin{cases}h_{i}, & i \text { odd }, \\ C_{i}, & i \text { even }\end{cases}
$$

generates a nonlinear algebraic system that must be solved at each time step. The IDA package provides a choice of Jacobian-free Newton-Krylov nonlinear solvers for this purpose. The termination criterion for the nonlinear iterations is based on ensuring

$$
\frac{\mathrm{R}}{1-\mathrm{R}}\|\delta \mathbf{y}\|<\sigma,
$$

where $\mathbf{R}$ is an estimate of the linear convergence rate and $\delta \mathbf{y}$ is the difference between successive iterates [5].

For the tolerance $\sigma$, IDA uses a default value of $\sigma=0.33$. However, Tocci et al. [8] found that $\sigma=0.033$ was a more appropriate value when 
solving Richards' equation. Our findings agree, with $\sigma=0.033$ found to be more efficient, in terms of the number of time steps required to compute solutions, than either $\sigma=0.33$ or $\sigma=0.0033$ (see Section 4.2). Hence, we take $\sigma=0.033$.

GMres is used for the underlying linear solver. Preconditioning is handled by the parallel band-block-diagonal preconditioner provided by IDA, which computes local, banded LU factorisations on each mesh subdomain (ignoring any ghost node contributions), thereby generating a block diagonal, inexact LU factorisation overall [5].

\section{Results}

We consider a test problem, and an application to the Bundaberg region of Queensland. Parameter values consistent across both problems are $C_{s}=$ 1.0 (non-dimensionalised), $\mathrm{g}=9.81 \mathrm{~m} \mathrm{~s}^{-2}, \alpha=1.0 \times 10^{-8} \mathrm{~m} \mathrm{~s}^{2} \mathrm{~kg}^{-1}, \beta=$ $4.47 \times 10^{-10} \mathrm{~m} \mathrm{~s}^{2} \mathrm{~kg}^{-1}, \rho_{0}=1000 \mathrm{~kg} \mathrm{~m}^{-3}, \rho_{\mathrm{s}}=1025 \mathrm{~kg} \mathrm{~m}^{-3}, \mu=1.0 \times$ $10^{-3} \mathrm{~kg} \mathrm{~m}^{-1} \mathrm{~s}^{-1}$ and $\mathrm{q}=0 \mathrm{~s}^{-1}$. Initial conditions are of hydrostatic head and fresh water.

\subsection{Test problem}

The test problem is one of seawater intrusion in a phreatic aquifer receiving recharge, taken from Huyakorn et al. [6], and illustrated schematically in Figure 1. Additional parameter values for this problem are $\phi=0.25, \mathrm{D}_{\mathrm{m}}=$ $0.0 \mathrm{~m}^{2} \mathrm{~s}^{-1}, \alpha_{\mathrm{L}}=10.0 \mathrm{~m}, \alpha_{\mathrm{T}}=5.0 \mathrm{~m}, \mathrm{U}_{\text {top }}=2.31 \times 10^{-8} \mathrm{~m} \mathrm{~s}^{-1}, \mathrm{u}_{\text {right }}=$ $4.63 \times 10^{-8} \mathrm{~m} \mathrm{~s}^{-1}$ and $\mathbf{k}=\operatorname{diag}(4.72,4.72,0.472) \times 10^{-12} \mathrm{~m}^{2}$. The steady state 0.5 isochlor is shown in Figure 2, which agrees well with the results published by Cheng et al. [4, Figure 6]. 


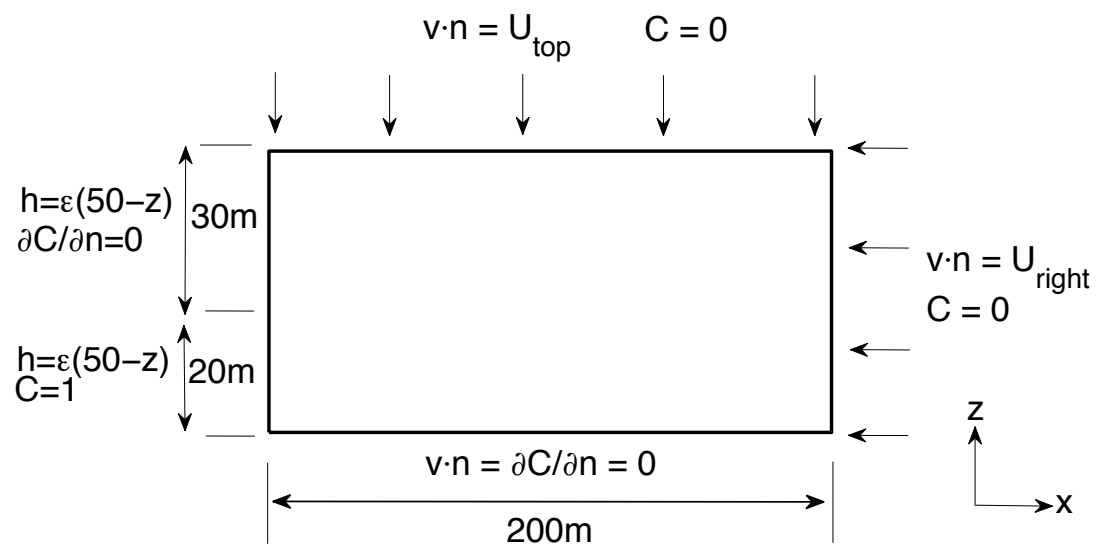

FiguRE 1: Test problem schematic.

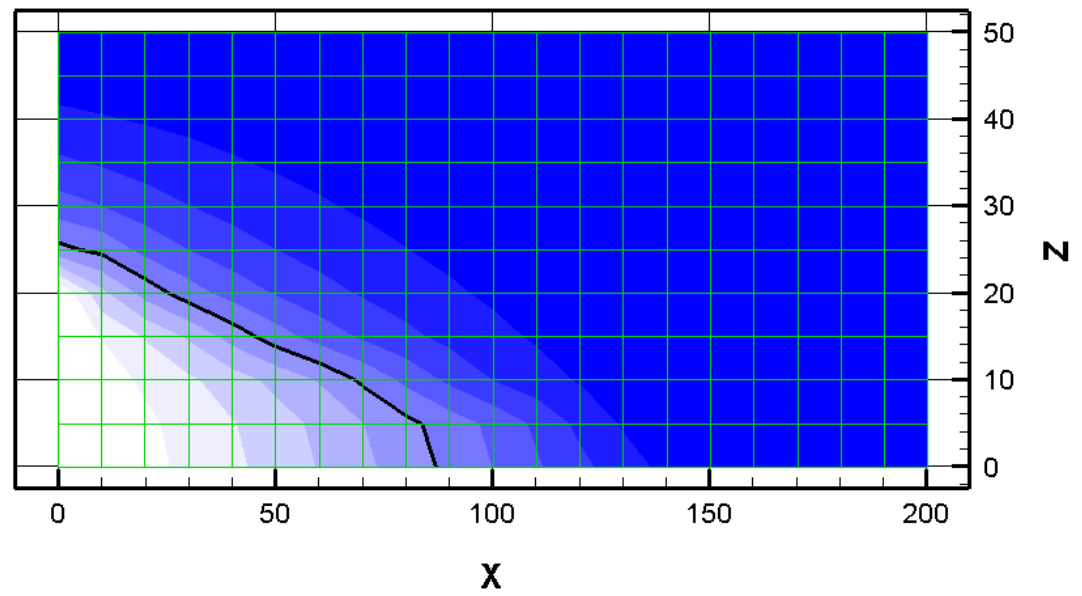

Figure 2: Steady state solution for test problem with 0.5 isochlor shown. 


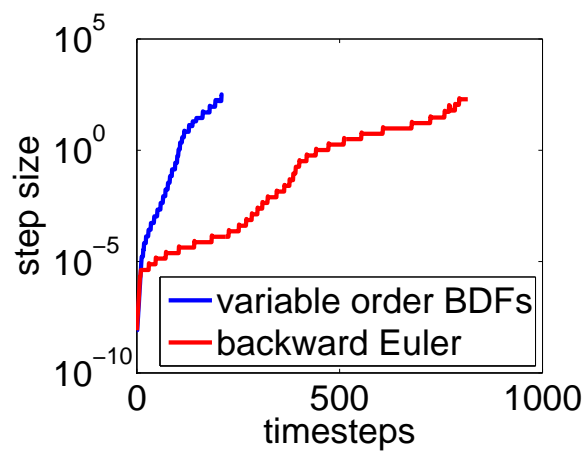

(a)

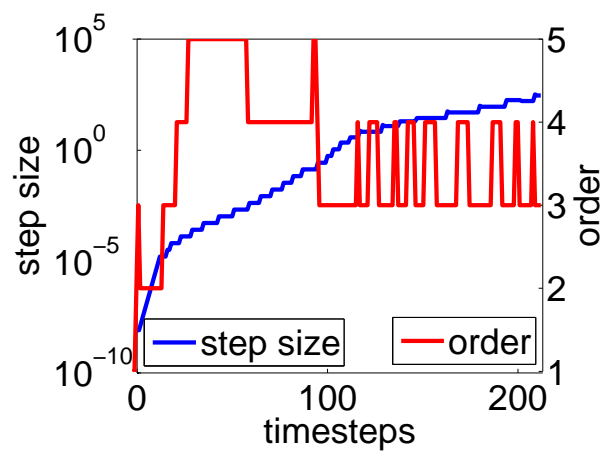

(b)

Figure 3: Step size versus number of time steps for test problem: (a) variable order BDFs and the backward Euler method; (b) variable order BDFs and their associated order.

Figure 3(a) compares the size of the time steps taken when using variable order BDFs, to those taken when using the backward Euler method (obtained by setting the maximum allowable order to be one in IDA). Using variable order BDFs leads to a significant reduction in the number of time steps required to advance the solution to its steady state. This was also reflected in the number of function evaluations required in the solution of the associated nonlinear algebraic system, with variable ordering BDFs requiring just 524 evaluations, compared to the 2080 evaluations required by the backward Euler method.

Figure 3(b) shows the order of the BDFs that were used at each step in the variable order case. The figure confirms that BDFs as high as fifth order are being utilised for the majority of the early time steps, while at later times the method settles for either third or fourth order, apparently unable to choose decisively between the two. 
Permeability (square metres)
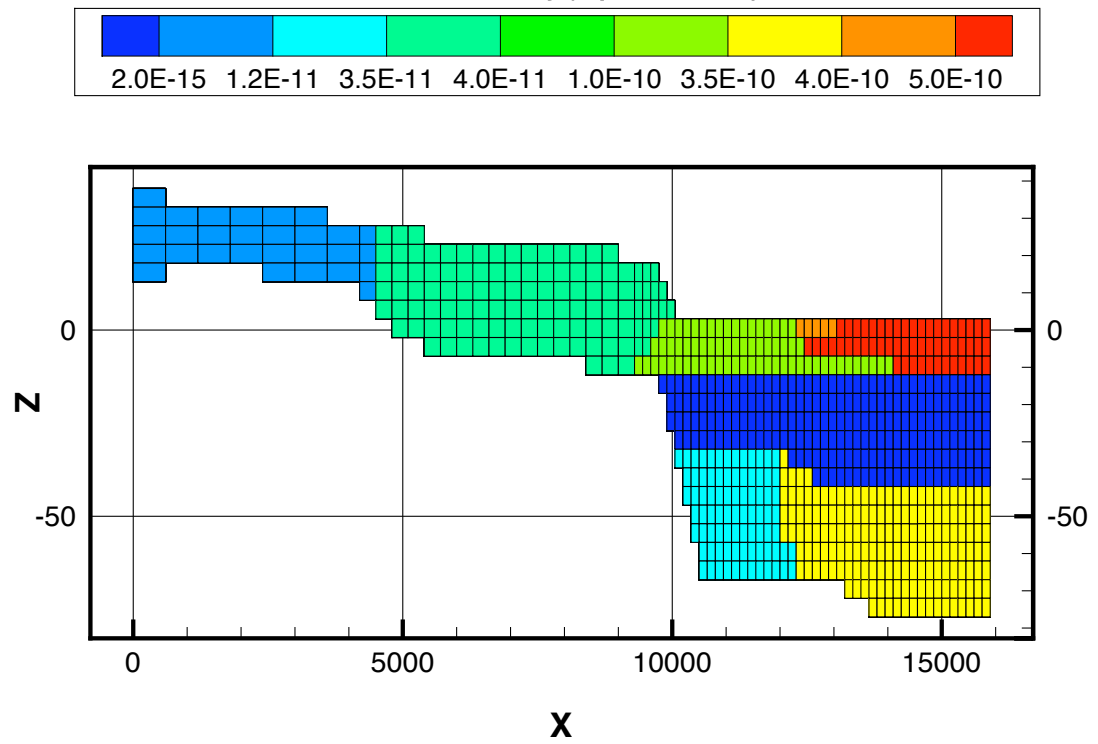

Figure 4: Permeabilities for the Bundaberg problem.

\subsection{Bundaberg problem}

For the second problem, we consider a cross section of the coastal alluvial aquifer system in the Bundaberg region of Queensland. This heterogeneous, anisotropic aquifer system comprises two aquifers separated by a leaky aquitard [1]. The mesh geometry, along with the permeabilities in the horizontal direction, are illustrated in Figure 4. Permeabilities in the vertical direction are estimated to be 1000 times less than in the horizontal [1].

The system receives recharge from rainfall along the top, at rates of $60 \mathrm{~mm}$ per year for the first $4.5 \mathrm{~km}$ and $90 \mathrm{~mm}$ per year for the next $9.6 \mathrm{~km}[1]$. We follow Zhang et al. [9] and impose equivalent freshwater heads and constant salt concentration for the remaining distance across the top, and along the seaward (right) boundary. The inland (left) and lower boundaries are treated 
as no-flow, being formed by relatively impermeable bedrock [1]. Remaining parameter values for this problem are $\phi=0.34, D_{m}=3.0 \times 10^{-9} \mathrm{~m}^{2} \mathrm{~s}^{-1}$, $\alpha_{\mathrm{L}}=100.0 \mathrm{~m}$ and $\alpha_{\mathrm{T}}=20.0 \mathrm{~m}$.

Although there is substantial groundwater extraction from this aquifer system, the problem must first be solved assuming no extraction in order to determine the initial condition for the full simulation [1]. It is this problem that we analyse here. Two solutions are shown in Figure 5: the solution after 100 years; and the steady state solution (which is after several thousand years). Both are in good agreement with the results published by Zhang et al. [9, Figures 8 and 10].

Figure 6(a) compares the size of the time steps taken to step to 100 years when using variable order BDFs, against those taken when using the backward Euler method. Once again the improved efficiency offered by the variable order scheme is apparent, with fewer than a third of the number of steps required compared to the backward Euler method.

Figure $6(\mathrm{~b})$ confirms the effectiveness of using $\sigma=0.033$ in (10), with this choice leading to faster, and less erratic progress towards 100 years, than either $\sigma=0.33$ or $\sigma=0.0033$.

\section{Conclusion}

The accurate simulation of saltwater intrusion in coastal aquifer systems is a difficult task. We used a method of lines approach incorporating a control volume, finite element, spatial discretisation, combined with variable order backward differentiation formulas, using the IDA package. With this approach, we demonstrated how the use of variable order BDFs leads to significantly improved efficiency in the time stepping process compared to traditional backward Euler approaches. This improved efficiency comes from the ability to use larger time steps while still maintaining accuracy in the 

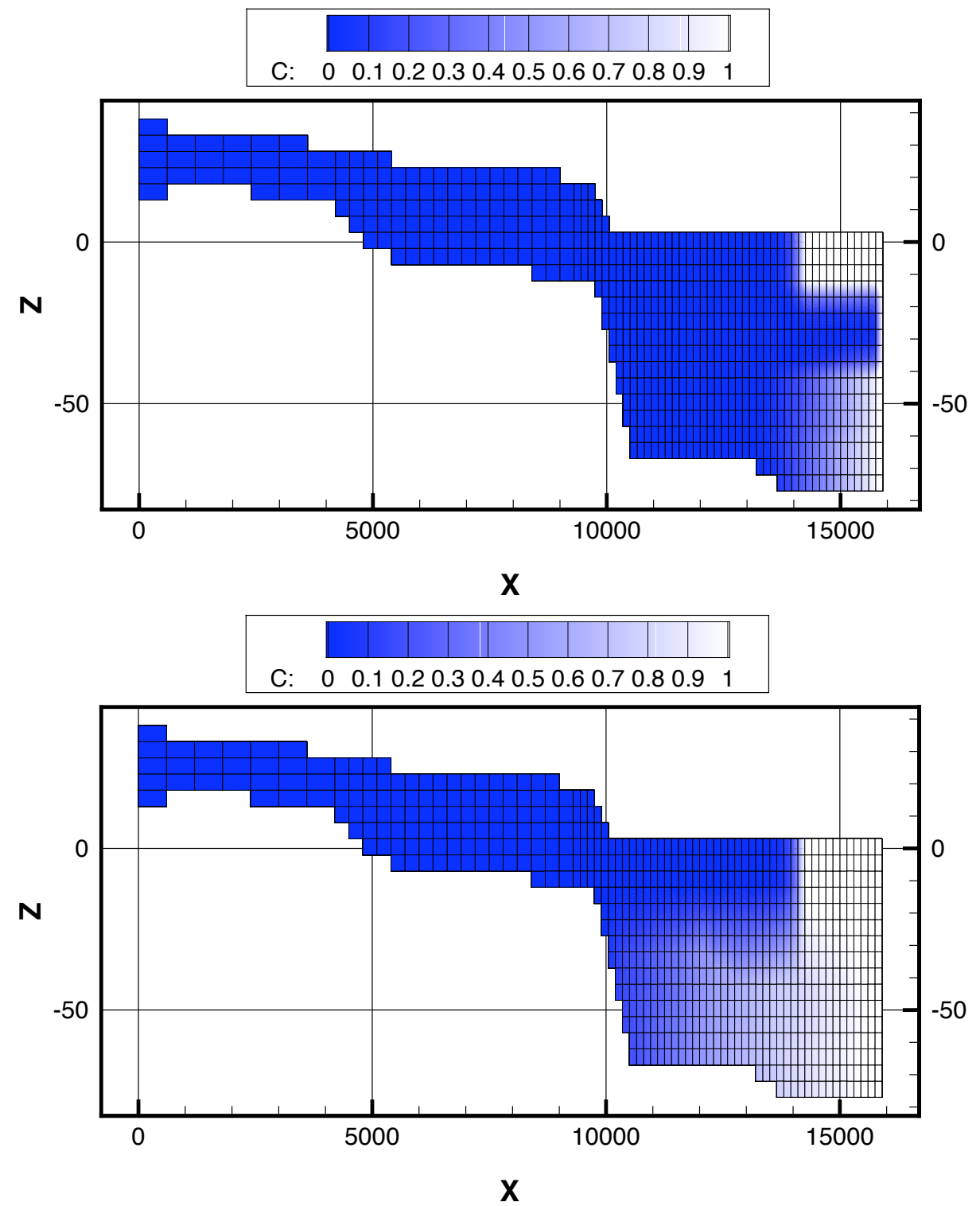

Figure 5: Solution to the Bundaberg problem at 100 years (top); steady state (bottom). 


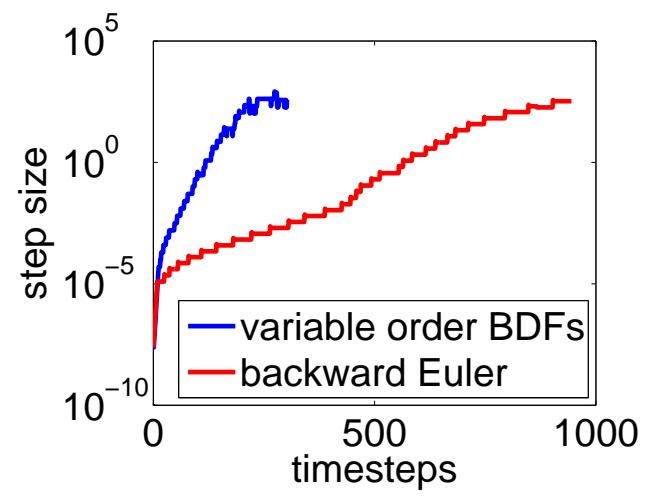

(a)

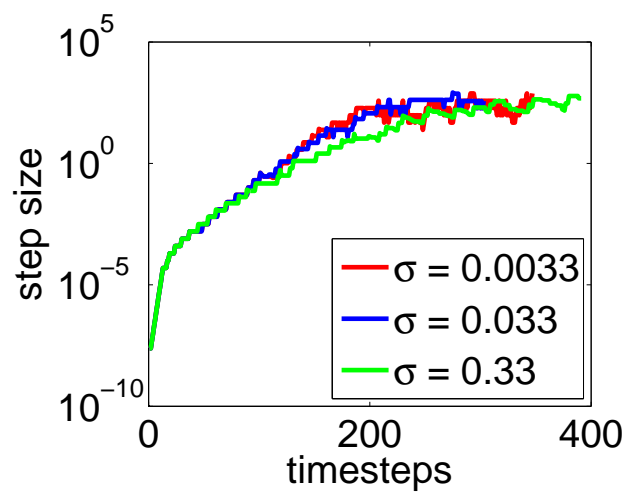

(b)

Figure 6: Step size versus number of time steps for Bundaberg problem: (a) variable order BDFs and the backward Euler method; (b) the effect of $\sigma$ on variable order BDFs.

computed solution, hence resulting in a greatly reduced number of time steps required to reach the final solution time.

\section{References}

[1] K. Bajracharya, J. Arunakumaren, and W. J. Huxley. Numerical modelling of seawater intrusion in Gooburrum, Bundaberg, Queensland. In T. R. Weaver and C. R. Lawrence, editors, Proceedings, Groundwater: Sustainable Solutions, pages 613-618. University of Melbourne, 1998. C626, C634, C635

[2] J. Bear. Hydraulics of groundwater. McGraw-Hill, New York, 1979. C627 
[3] K. E. Brenan, S. L. Campbell, and L. R. Petzold. Numerical solution of initial-value problems in differential-algebraic equations. SIAM, Philadelphia, 1996. C630

[4] Jing-Ru Cheng, Robert O. Strobl, Gour-Tsyh Yeh, Hsin-Chi Lin, and Woo Hee Choi. Modeling of 2D density-dependent flow and transport in the subsurface. J. Hydrologic Eng., 3(4):248-257, 1998. doi:10.1061/(ASCE)1084-0699(1998)3:4(248) C626, C631

[5] A. C. Hindmarsh, P. N. Brown, K. E. Grant, S. L. Lee, R. Serban, D. E. Shumaker, and C. S. Woodward. SUNDIALS: Suite of nonlinear and differential/algebraic equation solvers. ACM Transactions on Mathematical Software, 31(3):363-396, 2005. https: //computation.llnl.gov/casc/nsde/pubs/toms_sundials.pdf C630, C631

[6] P. S. Huyakorn, P. F. Andersen, J. W. Mercer, and Jr. H. O. White. Saltwater intrusion in aquifers: Development and testing of a three-dimensional, finite-element model. Water Resources Research, 23(2):293-312, 1987. C626, C627, C628, C631

[7] F. Liu, V. V. Anh, I. Turner, K. Bajracharya, W. J. Huxley, and N. Su. A finite volume simulation model for saturated-unsaturated flow and application to Gooburrum, Bundaberg, Queensland, Australia. Applied Mathematical Modelling, 30(4):352-366, 2006. doi:10.1016/j.apm.2005.05.007 C626, C628, C629

[8] M. D. Tocci, C. T. Kelley, and C. T. Miller. Accurate and economical solution of the pressure-head form of richards' equation by the method of lines. Advances In Water Resources, 20(1):1-14, 1997. doi:10.1016/S0309-1708(96)00008-5 C627, C630

[9] Q. Zhang, R. E. Volker, and D. A. Lockington. Numerical investigation of seawater intrusion at Gooburrum, Bundaberg, Queensland, Australia. 
Hydrogeology Journal, 12:674-687, 2004. doi:10.1007/s10040-004-0333-5 C626, C634, C635

\section{Author addresses}

1. T. J. Moroney, School of Mathematical Sciences, Queensland University of Technology, Brisbane, Australia.

mailto:t.moroney@qut.edu.au

2. S. L. Truscott, Department of Mathematics and Information Sciences, Tokyo Metropolitan University, Tokyo, JAPAN. mailto:truscott@ed.tmu.ac.jp 